\title{
Ion acceleration and positron production and annihilation in solar flares
}

\author{
Nidhal Guessoum ${ }^{1}$ \\ ${ }^{1}$ Physics Dept., American University of Sharjah, United Arab Emirates \\ email: nguessoum@aus.edu
}

\begin{abstract}
I first review: a) the current state of knowledge of ion acceleration in solar flares; b) the physics of positron production and annihilation; and c) recent RHESSI data on solar flare annihilation radiation. I then show how the modeling of the positron production and annihilation in the chromosphere, coupled with the newly available high-resolution data on the $511 \mathrm{keV}$ annihilation line, can have important physical implications w. r. t. the models: a) information on the temperature and density of the chromosphere; b) constraints on some of the physical characteristics of the flare and to some extent on the acceleration process.

Although I do mention past instruments (SMM and Yohkoh), this review focuses on the RHESSI satellite, considering the quantum leap it has constituted in the quality of the data it is providing and consequently the constraints it can place on models (of ion acceleration, annihilation environment, etc.).
\end{abstract}

Keywords. Sun: flares, acceleration of particles, line: profiles, gamma rays: theory, observation.

\section{Introduction: solar flare energy characteristics}

Solar flares release large amounts of energy, ranging from $10^{28}$ to $10^{34}$ ergs over timescales that vary from less than a second to almost an hour; a large part of that energy comes out in the form of energetic particles.

For high energy astrophysicists, flares are particularly interesting in the various forms of data that can be available in analyzing them. Among these tools, one can find: continuum emission over the entire electromagnetic range, i.e. from radio to gamma rays exceeding 1 $\mathrm{GeV}$; rich gamma-ray line emission from a few hundred $\mathrm{keV}$ to about ten $\mathrm{MeV}$; energetic particles, both charged and neutral, that can be detected in space as well as on Earth.

Flares are traditionally divided into two main categories: impulsive ones, which are compact and occur in the corona and exhibit marked ion enhancements, and gradual ones, which occur at greater heights, tend to correlate with coronal mass ejections, but have "normal" (ambient) ion compositions. Collisions between accelerated ions (with E $~$ $1 \mathrm{MeV} /$ nucleon) and ambient nuclei produce excited nuclei (which then emit characteristic gamma rays as lines) as well as pions (which then decay into high-energy photons or positrons that lead to observational effects), neutrons, and other particles. In addition to line emissions, gamma-ray production can occur by bremsstrahlung.

There are three major parts to solar flare phenomena: a) the acceleration processes, which determine the composition and energy distribution of particles; b) the transport/propagation of particles from one region to another; 3 ) the reactions and emission processes that result in the observed spectra. A large body of work, observational and theoretical (including numerical simulations) has been performed in each of these three sub-domains. In this short review, I will address the first and the third only; furthermore, I will limit the review of the acceleration stage to the ion species, the electrons undergoing different processes and energetics and leading to continuum emission that is not related to our main topic, i.e. positron production and annihilation in the flares. 
Ions have been observed (by interplanetary detectors) at energies up to $100 \mathrm{MeV}$ per nucleon (e.g. Reames et al. 1992) or more. This then explains the "bump" at around $70 \mathrm{MeV}$ that has been seen in several flare spectra, and which can only be attributed to neutron pion decays, the pions themselves having to come from the collision of very energetic protons (the pion production threshold being $\sim 300 \mathrm{MeV}$ ). The observation of neutrons in space and by ground-based detectors (at E between 50 and $500 \mathrm{MeV}$ ) indicates the acceleration of protons to energies up to $1 \mathrm{GeV}$ (Ramaty \& Mandzhavidze, 1994). Most flares confirm the acceleration of ions to $100 \mathrm{MeV}$ or so, but rare ones show exceptional acceleration of protons to between 1 and $10 \mathrm{GeV}$.

\section{Ion acceleration}

What is most striking is the fact that such accelerations are typically achieved over timescales of a few seconds or less; the rare and exceptionally strong ones take about 10 seconds to accelerate the protons to several GeV. As to the number of protons that are pumped in moderate and strong flares, a decade ago the estimate was that $10^{32}$ protons are propelled to energies greater than $30 \mathrm{MeV}$, which - depending on the particle spectrum one assumes (power law or Bessel function) - translates to about $5 \times 10^{35}$ protons of more than $1 \mathrm{MeV}$ and about $10^{37}$ protons of more than $20 \mathrm{keV}$. More recent work, however (Miller et al. 1997 and references therein), has led to an upward revision of this figure to $3 \times 10^{36}$. The energy content of ions in typical flares is now estimated at about $3 \times 10^{31}$ ergs, more than an order of magnitude larger than was believed a decade or so ago. This then compares about equally to the typical nonthermal electron energy and to the energy contained in a $1 \mathrm{kG}$ coronal field in a volume of $10^{27} \mathrm{~cm}^{3}$.

Acceleration processes have traditionally been split into 3 broad classes: stochastic acceleration by waves, shock acceleration, and direct electric field (DC) acceleration (Miller et al. 1997). These can be applied to both electrons and ions, and observational constraints can rule out some processes for the two groups of particles (leptons and hadrons). Indeed, one must pay particular attention to the following observables: the energy distributions of particles (which are either measured directly or linked to the resulting photon spectra); isotopic enhancements; acceleration time; etc. For instance, a major weakness of the DC electric field models is their inability to produce ions above even a few MeV. Not all acceleration models, however, are developed enough to allow for a direct confrontation with observations; such is the case, to a large extent, of shock and turbulent models. One can state in general terms that although all acceleration mechanisms play some role (Aschwanden 2002), MHD turbulence models present the most concordance with the constraining tests, with shocks presenting more uncertainties and unknown predictions, and DC models showing the most failures and disagreements with observations.

\section{Physics of flares revealed by gamma-ray spectroscopy}

Accelerated particles undergo nuclear reactions with nuclei of the ambient medium, producing excited or radioactive nuclei as well as pions when the available kinetic energies are high enough. The excited and the radioactive nuclei then produce characteristic gamma-ray lines and/or positrons, which go on to annihilate and produce a signature line at $511 \mathrm{keV}$. The observation, recording, and analysis of gamma-ray spectra can yield significant information on the temperature, density, and ionization state of the medium as well as on the accelerated particles' energy distributions; the more refined the recorded photon spectrum is, the more precise the inferred physical information will be, and that is why sensitive and high-resolution instruments are sought. 
The first spectroscopic measurements were performed in the seventies by balloons (Chupp et al. 1973) and the Solar Maximum Mission (SMM) satellite, which carried a low energy-resolution NaI spectrometer. The next gamma-ray satellites were Yohkoh and CGRO. The current satellite, the Reuven Ramaty High Energy Solar Spectroscopic Imager (RHESSI), has been recording solar gamma radiation with high spatial and energyresolution Ge detectors for the first time (Share et al. 2003) allowing a detailed physical investigation of high-energy solar phenomena.

It is not an exaggeration to state that RHESSI has constituted a quantum leap in the field. Indeed the high quality of the spectra and the images now obtained allow one to perform detailed diagnostics of flares, especially in analyzing the widths and profiles of gamma-ray lines, particularly the $511 \mathrm{keV}$ annihilation line, as well as local spatial explorations. Such analyses are capable of extracting a wealth of physical information about the flare regions, including temperature profile, density, ionization levels, energy spectrum and abundances of ions (both in the accelerated beams and in the ambient, target medium), and anisotropy (and thus acceleration and transport) of particles.

The many measurements performed to date allow one to make the following general observations: a) at least $30 \%$ of flares with emission at more than $300 \mathrm{keV}$ exhibit $\gamma$-ray line features; b) at least 15 de-excitation lines have been identified in flare spectra; c) although line widths could only be determined accurately with the recent Ge detectors of RHESSI, theoretical studies always predicted narrow nuclear lines.

Analyzing line spectra is also an important tool for determining accelerated particle distributions. Indeed, line ratios provide estimates of the spectral indices of flare-accelerated particles in different energy ranges: $6.13 \mathrm{MeV} / 1.63 \mathrm{MeV}$ lines are useful for the 2-20 $\mathrm{MeV} /$ nucleon particle spectra; $2.22 \mathrm{MeV} / 4.44 \mathrm{MeV}$ for the 10-100 MeV/nucleon range; and $0.511 \mathrm{MeV} / 4.44 \mathrm{MeV}$ for the $10-500 \mathrm{MeV} /$ nucleon range of particle energy distributions. Such measurements yield estimates of the energy contained in the accelerated ions. The $511 \mathrm{keV}$ (annihilation) line also provides important indications on pion production.

\section{Positron annihilation in solar flares in the RHESSI era}

Positrons are rather commonly produced in astrophysical environments; they normally result from the decay of pions and radioactive nuclei or from $\gamma-\gamma$ pair production. Positron production in solar flares was extensively investigated by Ramaty and co-workers from the mid-seventies to the mid-nineties (e.g. Ramaty et al. 1975; Kozlovsky et al. 1987). Indeed the topic was ignited by two seminal works: the early observation of the annihilation radiation from solar flares by Chupp et al. (1973), and the first comprehensive theoretical study by Crannell et al. (1976).

Positron processes in interstellar settings have been treated in detail by Guessoum et al. (2005, most recently), while the most extensive treatment for solar flare conditions was recently performed by Murphy et al. (2005). After being produced, positrons can annihilate "in flight" while slowing down or after thermalization with the ambient medium. Positronium (Ps), the bound state of a positron and an electron, can be formed, especially in a neutral medium, but Ps gets broken down at high temperatures and densities. The decay of Ps results in 2 or 3 photons, depending on the initial spin configuration of the system (parallel or anti-parallel); in the antiparallel-spin case, the decay results in a line, in the parallel-spin case, the photons have energies distributed continuously between 0 and $511 \mathrm{keV}$, resulting in a characteristic continuum "wing" in the spectrum. The width of the line and the continuum level yield information on temperature and density.

The major difference between the interstellar and the solar settings is that in solar flares, high densities $\left(\mathrm{n}_{\mathrm{H}} \sim 10^{15} \mathrm{~cm}^{-3}\right)$ as in the chromosphere, and temperatures above a 
few thousand degrees lead to the quenching of the ortho-positronium (parallel-spin) state and thus of the continuum wing, an important effect to include in spectral analyses in order to infer correct values for the density and temperature of the annihilating medium.

RHESSI has now made several high-quality observations of the $\mathrm{e}^{+} \mathrm{e}^{-}$annihilation line in solar flares: July 23, 2002; Oct 28 and Nov 2, 2003 (Share et al. 2002, Share et al. 2004, Smith et al. 2003). The analysis indicates that the line width varies during the flare, from somewhat broad $(\sim 4-8 \mathrm{keV})$ early in the event to rather narrow $(\sim 1-2 \mathrm{keV})$ within about 2 minutes; this, combined with the low levels of positronium continuum emission, indicates a dense annihilation medium $\left(\mathrm{n}_{\mathrm{H}}>10^{15} \mathrm{~cm}^{-3}\right)$.

Indeed, spectra can be theoretically constructed for various physical media (see Fig. 5 of Guessoum et al. 2005 and Fig. 20 of Murphy et al. 2005), where all processes and effects (from annihilation "in flight" by fast positrons to thermal processes, including positronium quenching in dense conditions) are taken into account; such theoretical spectra are then confronted with observed spectra, the best fit giving the medium's physical parameters. Analysis of the October and November 2003 flares by Share and coworkers indeed showed that the annihilate media can be multiple, ranging from hot $\left(\mathrm{T}=3--4 \times 10^{5} \mathrm{~K}\right)$ and ionized (when the line is broad) to cool $(\sim 5000 \mathrm{~K})$ and partially ionized (ionization fraction of at least $20 \%$ ).

\section{Conclusions}

Data analyses of RHESSI solar flare spectra have led to the general conclusion that the $511 \mathrm{keV}$ line mostly originates from positrons annihilating in densities $\gtrsim 10^{13} \mathrm{H}$ $\mathrm{cm}^{-3}$ and at temperatures above $10^{5} \mathrm{~K}$, except toward the end of the flare event when the line narrows significantly, indicating a cooler medium. This interpretation appears to challenge our current understanding of the solar atmosphere, even though there were earlier propositions for such high-density and high-temperature environments in flares (e.g. Doschek et al. 1977). More flares are being observed by RHESSI and analyzed at present, so more information on solar flare physics will be obtained soon ...

Moreover, RHESSI's imaging capabilities have indicated that the hard X-ray and gamma-ray emission tends to be relatively localized, which implies preferential sites of particle acceleration. Clearly the problem of ion acceleration is interrelated with the problem of gamma-ray production, not to mention the important issue of particle transport, so that a complete understanding of solar flares and the underlying magnetic topology is an ambitious objective, to which RHESSI is making contributions.

\section{References}

Aschwanden, M. J. 2002, Space Sci. Rev. 101, 1.

Chupp, E. L., Forrest, D. J., Higbie, P. R. et al. 1973, Nature 241, 333.

Crannell, C. J., Joyce, G., Ramaty, R., \& Werntz, C. 1976, ApJ 210, 582.

Doschek, G. A., Feldman, U., Rosenberg, F. D. 1977, ApJ 215, 329.

Guessoum, N., Jean, P., Gillard, W. 2005, A\& A 436, 171.

Kozlovsky, B., Lingenfelter, R. E., Ramaty, R. 1987, ApJ 316, 801.

Miller, J. A., Cargill, P. J., Emslie, A. et al. 1997, JGR 102, 14631.

Murphy, R. J., Share, G. H., Skibo, J. G., Kozlovsky, B. 2005, ApJS 161, 495.

Ramaty, R., Kozlovsky, B., Lingenfelter, R. E. 1975, SSRv 18, 341.

Reames, D. V., Richardson, I. V., Wenzel, K.-P. 1992, ApJ 387, 715.

Share, G. H., Murphy, R. J., Skibo, J. G. et al. 2003, ApJ 595, L85.

Share, G. H., Murphy, R. J., Smith, D. M. et al. 2004, ApJ 615, L172.

Smith, D. M., Share, G. H., Murphy, R. J. et al. 2003, ApJ 595, L81. 